\title{
Flexible real-time MIMO channel sounder for multidimensional polarimetric parameter estimation
}

\author{
P. Laly, D. P. Gaillot, M. Lienard, and P. Degauque \\ Institute of Electronics, Microelectronics and \\ Nanotechnology \\ IEMN/TELICE University of Lille \\ Villeneuve d'Ascq, France \\ Pierre.laly@univ-lille1.fr
}

\author{
E. Tanghe, W. Joseph, and L. Martens \\ Dept. of Information Technology \\ Ghent University \\ Ghent, Belgium \\ Wout.joseph@intec.ugent.be
}

\begin{abstract}
This paper describes the architecture of a fully parallel multiple-input multiple-output (MIMO) channel sounder. It has been designed to give in real-time the full polarimetric channel matrix which is then exploited to display, for example, the bi-directional channel characteristics as the angle of arrival (AoA) and angle of departure (AoD) of the multipath components. This $16 \times 16$ sounder, working at a center frequency of $1.35 \mathrm{GHz}$, uses an OFDM transmission scheme with an $80 \mathrm{MHz}$ bandwidth. Applications of this sounder are then illustrated by studying the outdoor to indoor propagation characteristics.
\end{abstract}

\section{Keywords-channel sounder; OFDM; MIMO; channel characteristics}

\section{INTRODUCTION}

Measurements of complex channel impulse responses are of prime importance to optimize the transmission scheme, either in indoor or outdoor environment, and to predict its performances. In the last decade numerous channel sounders have thus been developed as described in [1], [2], the transmitted signal occupying either a narrow band, a wide band [3] or even an ultrawide band as in [4]. Most of the sounders are multiple-input multiple-output (MIMO) systems in order to extract, from the measured channel transfer matrix, the double-directional characteristics of the link such as the angle of arrival (AoA), the angle of departure (AoD) and the relative time of arrival (ToA) of the multipath components.

The cheapest sounder is based on a sequential single-input single-output (SISO) architecture, the antenna elements being successively switched [5], [6]. Indeed, in this case, only a single transmitting $(\mathrm{Tx})$ and receiving $(\mathrm{Rx})$ module is needed. However, even if the switching time can be very fast, the time interval between two successive data recording must be greater than the delay spread of the channel. In outdoor environment and if the number of array elements is large, satisfying such a constraint may have a strong impact in terms of maximum Doppler frequency, channel coherence time and phase noise of the local oscillators as well, as outlined in [1]. To decrease the measurement time of a channel matrix, a first approach is to keep a switched transmitter but to implement a parallel channel receiver. In [7] for example, the chirp waveform transmitting signal at 1.9 and $2.1 \mathrm{GHz}$ and having a bandwidth of $60 \mathrm{MHz}$, is received on 8 channels, the Tx/Rx array elements being vertically polarized dipoles or patch antennas. Lastly a fully parallel transceiver architecture that employs a layered scheme of frequency and space-time division multiplexing has been proposed [8].

Whichever the sounding technique, a time consuming post-processing is usually needed to extract the channel parameters from the time domain samples of the received signal stored in a huge memory. Hence, It would be interesting to avoid such heavy processing in order to get in real-time the bi-directional channel characteristics which could be displayed while making the measurement campaign. To achieve this goal, a versatile channel sounder has been designed to reach an acquisition time less than $300 \mu$ s for a full polarimetric (16x16) channel transfer matrix $H . H$ is stored in a binary format to minimize the file size. Section II describes the main features of this sounder while Section III presents few examples dealing with outdoor-to-indoor scenarios.

\section{PRINCIPLE AND PERformanCES OF THE CHANNEl SOUNDER}

\section{A. Transmitted/Received Frames}

Let Ntx and Nrx be the number of array elements at Tx and $\mathrm{Rx}$, each element being a dual-polarized patch antenna. These elements can be placed in different ways to get a uniform linear or cylindrical array (ULA or UCA), or a rectangular array (URA). In our configuration, the maximum number of array elements being 8 , this leads to the measurement of a $16 \times 16$ channel matrix including both polarizations.

The total number Nsc of subcarriers of the OFDM scheme is 8192 occupying a total bandwidth of $100 \mathrm{MHz}$. However, for the time being, a 200 Msamples/s analog digital converter (ADC) is used at Rx such that the transmitting band has been reduced to $80 \mathrm{MHz}$. This is performed by not feeding subcarriers situated in the lower and higher frequency band of the spectrum. Newman phases are added to the default multitone to minimize the peak-to-average power ratio. Since the size of the Tx array can be chosen, the subcarriers can be allocated either to only one patch antenna or distributed among all $\mathrm{Tx}$ antennas. As an example with $\mathrm{Ntx}=8$, Fig. 1 shows the distribution of the 1024 subcarriers per antenna, their spacing being equal to $97.66 \mathrm{kHz}$. To keep reasonable 


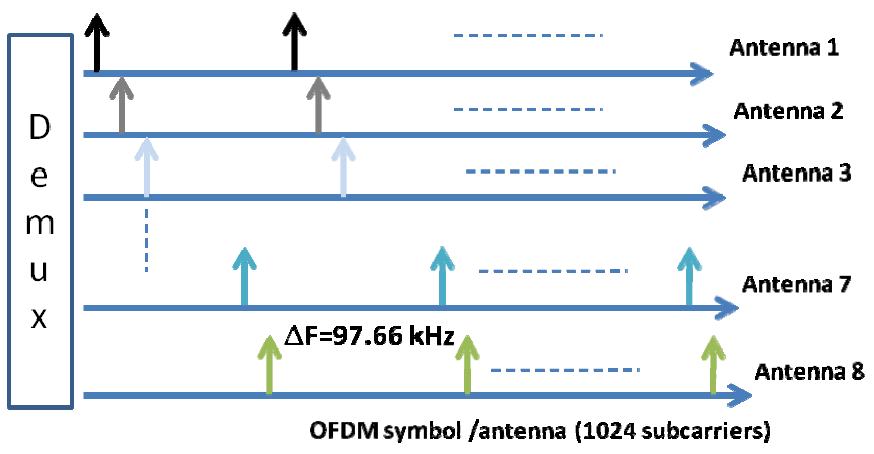

Fig. 1. Example of subcarriers allocation between 8 transmitting antennas

the global cost of the system, a switch with $50 \mathrm{~dB}$ isolation is used to change the polarization of each transmitting antenna to avoid doubling the RF front end. Therefore, 2 OFDM symbols are successively sent, the first one for one polarization and the other one for the orthogonal polarization. On the contrary, at $\mathrm{Rx}$, the signals measured at each of the 2 outputs of each bipolarized patch antenna are simultaneously stored. With a symbol duration of $\sim 82 \mu \mathrm{s}$ and by taking the duration of the preamble and of the cyclic prefix into account, the acquisition time of a full polarimetric channel matrix (deduced from the reception of 2 successive symbols) is about $300 \mu \mathrm{s}$.

Also, it must be outlined that the frame structure can be easily modified, as well as the preamble waveforms and the OFDM symbols, the length of the cyclic prefix and the number of symbols within a frame. For instance, one can choose to send only 2 symbols or, for example, 16 successive symbols or more. For the latter, the signal can be averaged or, on the contrary, the channel coherence time and Doppler information can be extracted from the variation of the channel transfer function.

\section{B. Main Features of the Hardware}

The baseband Tx module consists in 4 FPGA-400 MHz cards, each one including two 500 Msample/s 16-bit D/A converters and 1 Go RAM. Each of the 2 outputs of an FPGA controls 1 antenna. To avoid I/Q impairment, a real digital baseband signal is transmitted to the RF chain, despite the fact that the sampling rate of the digital to analog converter must be 2 times higher (200 Msample/s) than for an I/Q scheme. The RF signals are sent to each antenna after being processed in the RF filters and mixers.

At the receiving side, the signal processing module includes 2 FPGA cards with eight 200 MSample/s converters, each FPGA simultaneously processing 4 dual-port antennas. In the FPGAs, an OFDM symbol synchronization algorithm is applied to the received preamble and FFTs are performed in parallel. All modules are synchronized to a $10 \mathrm{MHz}$ rubidium clock. With such architecture, it must be outlined that no post processing is needed and the binary format files related to the $H$ MIMO channel matrix can be directly used for extracting the channel characteristics. Each element of $H$ is of course corrected by the gain of the automatic gain control and by the correction factors of the transmitting and receiving chains measured during a calibration procedure.

This work was supported in part by a France-Belgium INTERREG contract, by the Region Nord-Pas de Calais and by the French Ministry of Research (CISIT project)

\section{Antenna Characteristics}

The elementary dual-polarized patch antenna was designed with CST Microwave Studio software. The length and width of the ground plane is $103 \mathrm{~mm}$, the dimension of the metallic patch being about 1.4 times smaller. To obtain a wide band at $1.35 \mathrm{GHz}$, a 4-layer stacked configuration was chosen. The thickness of each layer, having a permittivity of 1.96 , is $2.5 \mathrm{~mm}$ and thin adhesive layers are placed between them. This leads to a total thickness of $10.4 \mathrm{~mm}$. The measured bandwidth, for a $\mathrm{S} 11$ of $-10 \mathrm{~dB}$, is $75 \mathrm{MHz}$, whereas the port isolation and cross polarization are larger than $30 \mathrm{~dB}$ and 25 $\mathrm{dB}$ across the whole frequency band, respectively.

For the ULA and URA, the distance between adjacent patches is a few $\mathrm{mm}$. For the UCA, the 8 patch antennas are equally spaced on the surface of a cylinder having a radius of $22 \mathrm{~cm}$., i.e. one wavelength at $1.35 \mathrm{GHz}$. Antenna array calibration is of course needed; results of such a calibration being used for AoA and AoD estimation.

\section{Extraction of the Bi-Directional Channel Characteritics and Performances of the Global System}

Some channel characteristics such as path loss, delay spread, channel stationary time and cross-polar discrimination factor (XPD) can be directly deduced from $H$. However, additional information on AoA, AoD and ToA of the multipath components is often needed to optimize the transmission scheme. The high-resolution algorithm (HRA) RiMAX [9] has been implemented. This parametric estimator relies on the observation that the probability density function of a radio channel follows a multivariate normal distribution. The deterministic part of the radio channel (i.e. mean of the distribution) solely depends on the specular components characteristics whereas the stochastic part (i.e. covariance) depends on the dense multipath components characteristics (diffuse scattering plus weak specular components). A joint deterministic/stochastic maximum likelihood estimation of the specular and dense multipath components is performed to retrieve the estimates of both propagation mechanisms. This approach was shown to increase the accuracy of the estimated specular components compared to other estimators wherein only additive white Gaussian noise is considered [10].

During the initialization phase of the HRA, a coarse estimation, yet close to the real solution, of the path characteristics is obtained in less than $300 \mathrm{~ms}$. Typically, the HRA takes about few minutes for a run on a laptop to extract the characteristics of about 20 paths. In conclusion, the main features of the channel sounder are:

- Up to 8 transmitting channels for a single polarization and to 16 by using a switch on dual-polarized antennas

- Up to16 simultaneous receiving channels

- Center frequency: $1.35 \mathrm{GHz}$, useful bandwidth: 80 $\mathrm{MHz}$, maximum number of subcarriers: 8192

- Any OFDM symbols can be uploaded

- Acquisition time of a 16x16 channel matrix: $300 \mu \mathrm{s}$

- No additional post processing is needed, the $H$ matrices in binary format being directly stored. 
- During on-site measurement campaigns, coarse estimation of DoA, DoD and delays can be displayed every $300 \mathrm{~ms}$.

- The power delay profiles can also be dynamically displayed every few ms

\section{Application to Outdoor to Indoor Propagation}

Previous published measurements on outdoor to indoor penetration into buildings are mainly related to the additional path loss, as described in [11]. In this case, the objective is to make a statistical analysis for different types of building and to propose path loss models. However to optimize diversity schemes and for MIMO applications as well, it is also important to measure the full polarimetric parameters of the bi-directional link. Rather than to study the penetration of a wave transmitted by a distant cellular base station, we have preferred to put a Tx array in the vicinity of the building in which the Rx array is situated, in order to manage the polarization of the wave incident on the building.

During measurements, the ULA Tx array was placed at a height of $7 \mathrm{~m}$ and at a distance of $50 \mathrm{~m}$ of the building. The main lobe of each patch array element is oriented towards the building. The Rx array, situated at the first floor of the building, i.e. at the same height as Tx, is moved in different rooms. This allows making a statistical analysis of the distribution of the channel characteristics and to point out the influence of the antenna polarization. In the following, in order to briefly illustrate results of the channel sounding, only 2 extreme cases are presented. The array is situated in the center of a room whose windows are either facing the Tx array (light indoor -LI-), or in a room on the other side of the building (deep indoor - DI-).
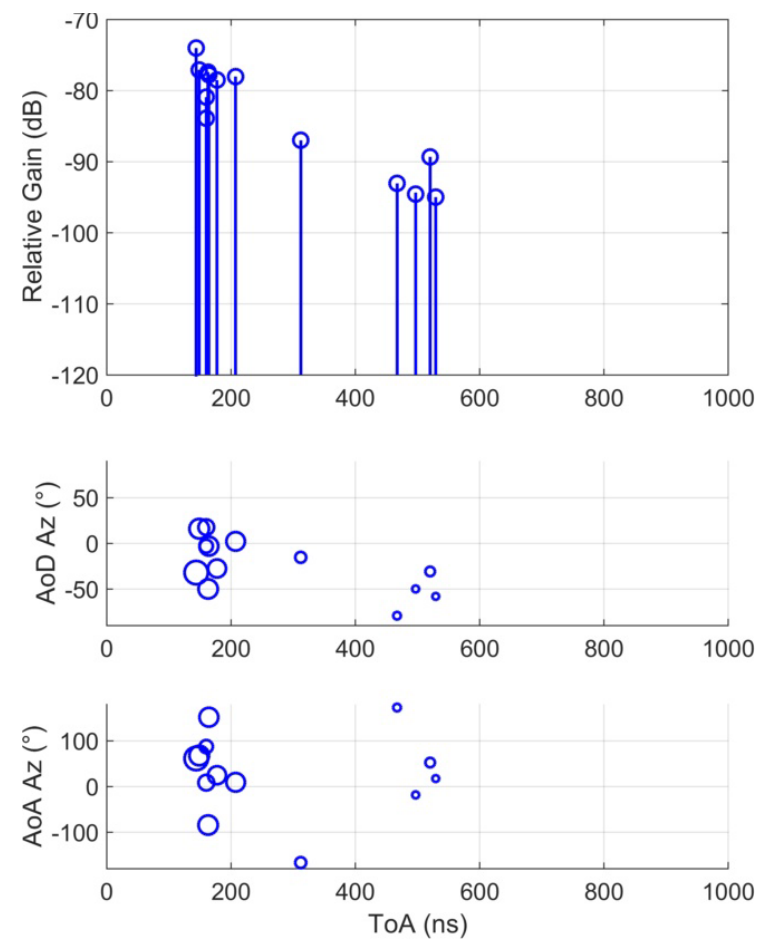

Fig. 2. AoA, AoD and ToA for a deep indoor scenario
For a DI scenario, Fig. 2 shows results obtained from RiMAX, i.e., a channel impulse response and the AoA/AoD associated with their relative ToA, thus referred to an arbitrary reference time, Tx and Rx being not synchronized. For this DI scenario the angular spread $\mathrm{AS}=80^{\circ}$ and the waves are nearly depolarized, the mean XPD factor being equal to $6 \mathrm{~dB}$. For the LI configuration, AS is much smaller $\left(10^{\circ}\right)$ and the waves remain polarized (Mean XPD $=15 \mathrm{~dB}$ ). The polarization, horizontal or vertical, of the incident plane wave has nearly no impact on these results. The XPD factor can also be calculated for each multipath component. Results are given in Table I for 3 paths. For the DI scenario, XPD does not vary appreciably while, for LI, XPD strongly decreases with the path delay.

TABLE I. XPD FOR A FEW PATHS

\begin{tabular}{|c|c|c|c|}
\hline \multicolumn{2}{|c|}{ DEEP INDOOR } & \multicolumn{2}{c|}{ LIGHT INDOOR } \\
\hline RELATIVE TOA & $\boldsymbol{X P D}(\boldsymbol{D B})$ & RELATIVE TOA & $\boldsymbol{X P D}(\boldsymbol{D B})$ \\
\hline 0 & 7 & 0 & 24 \\
\hline 17 & 10 & 15 & 10 \\
\hline 45 & 5 & 40 & 4 \\
\hline
\end{tabular}

\section{References}

[1] D. Laurenson and P. Grant, "A review of radio channel sounding techniques", Proc. of the $14^{\text {th }}$ European Signal Processing Conf., Florence, Italy, Sept. 4-8, 2006

[2] N. Czink, "Channel measurements", in Pervasive mobile and ambient wireless communications: COST action 2100, ch. I, R. Verdone and A. Zanella, Eds, Springer-Verlag, London, 2012, pp. 5-66

[3] F. Kaltenberger, M. Kountouris, L. S. Cardoso, R. Knopp, and D. Gesbert, "Capacity of linear multi-user MIMO precoding schemes with measured channel data", Proc. IEEE Intl. Workshop on Signal Processing Advances in Wireless Commun., Recife, Brazil, Dec. 2008

[4] R. Zetik, M. Kmec, J. Sachs, and R. S. Thomä, "Real-Time MIMO Channel Sounder for Emulation of Distributed Ultrawideband Systems", Int. J. of Antennas and Propag., Article ID 317683, 16 pages, 2014

[5] J. Kivinen, T. O. Korhonen, P. Aikio, R. Gruber and P. Vainikainen, "Wideband Radio Channel Measurement System at 2GHz," IEEE Trans. on Instrumentation and Measurement, vol. 48, pp. 39-44, Feb. 1999

[6] R. S. Thomä, D. Hampicke, A. Richter, G. Sommerkorn, and U. Trautwein, "MIMO vector channel sounder measurement for smart antenna system evaluation," European Trans. on Telecom., vol. 12, no. 5, pp. 427-438, 2001.

[7] S. Salous, R. Lewenz, I. Hawkins, N. Razavi-Ghods and M. Abdallah, "Parallel receiver channel sounder for spatial and MIMO characterisation of the mobile radio channel," IEE Proceedings on Commun., vol.152, pp. 912-918, Dec. 2005.

[8] M. Kim, J. I. Takada, and Y. Konishi, "Novel scalable MIMO channel sounding technique and measurement accuracy evaluation with transceiver impairments", IEEE. Trans. on Instrumentation and Measurement, vol. 61, no 12, pp. 3185-3197, Dec. 2012

[9] A. Richter, "Estimation of radio channel parameters: models and algorithms," Thesis, Depart. of Electric. Eng. and Inform. Technol., TU, Ilmenau, Germany, 2005.

[10] E. Tanghe, D.P. Gaillot, W. Joseph, M. Lienard, P. Degauque, L. Martens, "Robustness of high-resolution channel parameter estimators in presence of dense multipath components", Electron. Lett., vol. 48, no. 2, 130-132, 2012.

[11] Y. Hirota, H. Izumikawa and C. Ono, "Outdoor-to-indoor radio propagation characteristics with $800 \mathrm{MHz}$ band in an urban environment," in Proc. IEEE Antennas Propagation/URSI Symp., pp. 697-698., 2014 